\title{
La relación entre la espiritualidad en el ambiente laboral y el compromiso organizacional en un grupo de empleados de un municipio del área oeste de Puerto Rico ${ }^{1}$
}

Rita Azarell Vélez-Alvarado

Ph. D. Industrial \& Organizational Psychology Pontificia Universidad Católica de Puerto Rico

Correo electrónico: ritavelez@pucpr.edu

Roberto González Valles

PhD. Psicología Industrial Organizacional Pontificia Universidad Católica de Puerto Rico

\section{Resumen}

Objetivo: determinar la relación entre la espiritualidad en el ambiente laboral y el compromiso organizacional. Método: estudio descriptivo correlacional, la muestra estuvo conformada por 156 empleados del oeste de Puerto Rico. Como instrumento se utilizaron las sub-escalas de la dimensión de la espiritualidad en la unidad de trabajo y la organización de la escala de espiritualidad en el lugar de trabajo de Ashmos y Duchon y la escala de compromiso organizacional de Rosario-Hernández y Rovira-Millán. Resultados: existe relación significativa de manera inversa entre la espiritualidad en el lugar de trabajo y el compromiso organizacional; sin embargo, no se encontraron diferencias significativas entre los años de servicio y la espiritualidad en el lugar de trabajo. Conclusión: la relación inversa entre la espiritualidad en el lugar de trabajo y el compromiso organizacional resulta como consecuencia de la percepción negativa de los empleados ante las nuevas políticas organizacionales en Puerto Rico.

Palabras clave Espiritualidad en el lugar de trabajo, compromiso organizacional.

1 Para citar este artículo: Vélez-Alvarado, R. A., \& González, R. (2019). La relación entre la espiritualidad en el ambiente laboral y el compromiso organizacional en un grupo de empleados de un municipio del área oeste de Puerto Rico. Informes Psicológicos, 19(1), pp. 11-30 http://dx.doi.org/10.18566/infpsic.v19n1a01 


\section{The relationship between spirituality in the workplace and organizational commitment in a group of employees of a municipality in the western area of Puerto Rico}

Abstract

Objective: to determine the relationship between spirituality in the workplace and organizational commitment. Method: descriptive correlational study, with a sample of 156 employees from the west of Puerto Rico. The sub-scales of the dimension of spirituality in the work unit and the organization of the scale of spirituality in the workplace of Ashmos and Duchon, and the scale of organizational commitment of Rosario-Hernández and Rovira-Millán were used. Results: there is an inverse significant relationship between spirituality in the workplace and organizational commitment; however, no significant differences were found between years of service and spirituality in the workplace. Conclusion: the inverse relationship between spirituality in the workplace and organizational commitment results from the negative perception of employees before the new organizational policies in Puerto Rico.

Keywords

Spirituality in the workplace, organizational commitment.

\section{A relação entre espiritualidade no ambiente de trabalho e comprometimento organizacional em um grupo de funcionários de um município da região oeste de Porto Rico}

Resumo

Objetivo: determinar a relação entre espiritualidade no ambiente de trabalho e comprometimento organizacional. Método: estudo descritivo correlacional, a amostra foi composta por 156 funcionários do oeste de Porto Rico. Como instrumento foram usadas subescalas da dimensão da espiritualidade na unidade de trabalho e na organização da escala de espiritualidade no local de trabalho de Ashmos e Duchon assim como a escala de compromisso organizacional, porém não foram achadas diferencias significativas entre os anos de serviço e a espiritualidade no local de trabalho. Conclusão: a relação inversa entre a espiritualidade no local de trabalho e 0 compromisso organizacional vem como consequência da perceção negativa dos funcionários em face das novas politicas organizacionais no Porto Rico.

Palavras chave Espiritualidade no local de trabalho, comprometimento organizacional. 


\section{ntroducción}

La espiritualidad en el lugar de trabajo, de acuerdo con Marques, Dhiman y King (2005), es un fenómeno que no debe ser ignorado por la sociedad ni las organizaciones a fin de poder enfrentar los retos actuales y futuros. Cada día el ambiente laboral se torna más pequeño debido a los avances de la tecnología, e.g., internet, teléfonos celulares, video conferencias, así como la tendencia de las corporaciones a reducir sus instalaciones físicas (Heinsohn, 2012). Estos cambios han provocado un aumento en el interés de la espiritualidad en el lugar de trabajo debido a despidos masivos, aumento en la carga de trabajo, el estrés laboral, la eliminación del contrato psicológico, el uso de la internet y la tecnología (Fry, Vitucci, \& Cedillo, 2005; Heinsohn, 2012). Es en tiempos de vicisitudes que principalmente las personas recurren a la espiritualidad como fuente de energía (Marques et al., 2005; 2007). A pesar de que la espiritualidad es una experiencia interior muy personal, el concepto se ha comenzado a estudiar gracias a los beneficios que ésta proporciona en el desempeño y compromiso normativo a nivel organizacional (Ashmos \& Duchon, 2000; Jurkiewicz \& Giacalone, 2004; Marques et al., 2005).

Ashmos y Duchon (2000) señalan que el concepto de la espiritualidad representa la esencia necesaria para energizar la vida, los pensamientos y las acciones. Asimismo, constituye la creencia en el poder operacional que se deriva de la conectividad con todas las criaturas vivientes, es decir, la naturaleza, y la conciencia del propósito y significado de la vida (Young \& Koopsen, 2011). Con el advenimiento del siglo XXI, el concepto de la espiritualidad en el ambiente laboral ha sido un tema público y de concientización profesional; ha adquirido gran interés y cobertura entre las organizaciones, la política empresarial y la academia. Este paradigma tuvo sus orígenes a raíz de los atentados del 11 de septiembre del 2001 en la ciudad de Nueva York y el Pentágono, denominados comúnmente "El 9/11", y como consecuencia de la caída de los mercados en la bolsa de valores y la falta de integridad observada en las organizaciones. Dadas estas situaciones, las personas comenzaron a ser más conscientes sobre el valor humano y a experimentar un profundo sentido de compromiso hacia el lugar de trabajo (Marques et al., 2007).

De acuerdo con O'Brian (2014) y Young y Koopsen (2011), ésta tiene sus raíces en los valores espirituales y creencias religiosas. Los sociólogos han observado una creciente integración entre la religión y la espiritualidad en la vida profesional de las personas (Carroll, Stewart, \& Thompson, 2014). Las investigaciones han demostrado que existe una conexión entre la espiritualidad en el lugar de trabajo y los valores de la organización y con el comportamiento y la conducta ética, lo que permite alcanzar las metas y objetivos de la organización, satisfaciendo, a su vez, las necesidades del empleado. Por lo tanto, la conceptualización de la espiritualidad en el trabajo debe incluir no solamente el reconocimiento que establece que los individuos tienen vidas interiores que los mueven hacia la búsqueda de sentido, sino también la búsqueda de significado en el trabajo (Duchon \& Plowman, 2005; Jurkiewicz \& Giacalone, 2004; Maheshwari, 2015; Mitroff \& Denton,1999). 


\section{Espiritualidad en el ambiente laboral}

La espiritualidad en el ambiente de trabajo, de acuerdo con Marques et al. (2005), se refiere al marco de los valores de la organización, los cuales juegan un papel importante en la creación de la cultura organizacional y el clima laboral que debe permear en la rutina del trabajo. De acuerdo con Belalcázar (2012), el clima laboral son aquellas expectativas y demandas que los trabajadores que laboran en una organización tienen en torno a ella y al nivel de satisfacción de las mismas conforme al funcionamiento del lugar de trabajo. Mientras, la cultura la considera como el sistema de valores que está presente y conforma la organización y del cual se desprenden las normas y las pautas de conducta con los que las personas se sienten más o menos identificados. La espiritualidad provee sentido de paz y esparcimiento en el desempeño laboral y aporta al empleado sentido de responsabilidad por el trabajo que realiza, así como empatía hacia los demás, creando así una cultura de trabajo y trascendencia en la ejecución del mismo; produciendo, por ende, sentimientos de plenitud y alegría, así como sentimientos y pensamientos altamente refinados en los empleados (Marques et al., 2007).

En contraste, Hodge (2001) define la espiritualidad como una relación con Dios o una entidad que se considere superior, que provee significado, propósito y misión de vida. Esta relación produce estados afectivos tales como: altruismo, amor y el perdón, los cuales tienen un efecto significativo en las relaciones del individuo consigo mismo, con las otras personas, con la naturaleza y con lo que él considere como entidad superior. De acuerdo con Ashmos y Duchon (2000), la espiritualidad en el entorno laboral constituye la fortaleza central en el desempeño, el proceso de ayuda y la mediación de conflictos, y brinda a los individuos la capacidad de relacionarse y comunicarse.

Van der Walt y de Klerk (2014) explican que la espiritualidad envuelve la búsqueda del significado de la vida, a través de la cual se comunica un sentido de interconexión con uno mismo y los demás, manifestado por un continuo descubrimiento del sentido de la vida y de propósito personal. Igualmente, la introspección en torno a cuestionamientos continuos sobre quién se es realmente, qué se desea de la vida y qué legado se dejará. Estos cuestionamientos se convierten en el fundamento para una confrontación y exploración personal profunda, la cual trastoca la visión básica sobre la vida.

Kazemipour et al. (2012) sostienen que existe una gama de interpretaciones en torno a las explicaciones en la literatura sobre el tema de la espiritualidad, las cuales pueden agruparse en tres dimensiones: intrínseca, religiosa y existencial. La dimensión intrínseca alude a que la espiritualidad es un concepto o principio que se origina en el interior del individuo. Esta dimensión sustenta que ésta no se limita a los dogmas de la religión, pues tiene la capacidad de trascenderla, sino que abarca un sentimiento de estar interconectado con uno mismo, con los demás y la naturaleza. En síntesis, en la dimensión intrínseca, la espiritualidad se entiende como la búsqueda interna del significado y el sentido de la vida de cualquier individuo, independientemente de si pertenece o no a una denominación o afiliación religiosa (Gupta, Kumar, \& Singh, 2014). 
La dimensión religiosa de la espiritualidad, de acuerdo con Kazemipour et al. (2012), es aquella que surge de las creencias y entidades asociadas a una afiliación religiosa. Ésta se basa en la creencia de que existe un Ser Superior que brinda dirección a las personas, dotándolas de normas, ritos y prácticas sobre la armonía, la honestidad y la ética. La dimensión espiritual se engrandece según el individuo practica los ritos religiosos o normas establecidas por la religión a la que pertenece. Finalmente, la dimensión existencial hace mayor énfasis en la búsqueda del significado y propósito de la vida, y se manifiesta en todos los contextos sociales en que las personas interactúan. En esta dimensión se involucran la firmeza de los valores, los principios éticos y morales de la persona, así como su interrelación con las demandas del mundo exterior.

En esa misma dirección, Carroll et al. (2014) explican que los científicos sociales han observado una creciente integración entre la religión y la espiritualidad en la vida profesional de las personas. Los autores afirman que las pocas investigaciones realizadas en torno al tema han demostrado que las creencias espirituales influyen en el compromiso con el trabajo e incrementan la satisfacción intrínseca con el compromiso laboral. De acuerdo con varios autores, la espiritualidad representa las creencias y valores que tiene el ser humano (Fry et al., 2005; Gupta et al., 2014; Jurkiewicz \& Giacalone, 2004; Mitroff \& Denton, 1999).

Mitroff y Denton (1999) exponen que el ideal de la espiritualidad en el lugar de trabajo está impulsado por la interacción de factores que promueven el respeto hacia el prójimo, las actitudes y la visión de la organización. De este modo, no sólo se obtienen resultados positivos en el propio ambiente de trabajo, sino también, se traduce en el fortalecimiento del compromiso organizacional. La espiritualidad en el lugar de trabajo constituye un tema para la discusión, dado que empíricamente se ha probado una conexión entre el compromiso organizacional y la espiritualidad. Sin embargo, la religiosidad es considerada un concepto más cerrado, rígido e inapropiado para la discusión en ese ámbito (Jurkiewicz \& Giacalone, 2004; Madden, 2015); tampoco existe evidencia clara que conecte la religión con la ética (Gupta et al., 2014; Hodge, 2001).

\section{Compromiso organizacional}

El compromiso organizacional se describe como el nivel de importancia del trabajo en la imagen personal y el nivel de participación en el trabajo, en el cual la autoestima es afectada dependiendo de cómo el empleado percibe su rendimiento (French \& Kahn, 1962) y el nivel de importancia del trabajo en la vida privada del empleado, así como cuánto se identifica el empleado psicológicamente con su empleo (Gómez-Mejía, 1992). Debido a que los cambios y la métrica financiera tienen un rol decisional cada vez más importante, Kolinsky et al. (2008) explican que los líderes esperan que los empleados hagan lo que sea necesario a fin de mantener la productividad y afecten positivamente la organización. Para muchos trabajadores, esa dinámica significa que el trabajo tiene un lugar muy importante y les consume mucho tiempo en sus vidas. Como resultado, hay gran expectativa de parte de los empleados de satisfacer las necesidades de conectividad, significado, 
propósito, altruismo, virtud, sustento y esperanza en el lugar de trabajo.

Rosario-Hernández y Rovira-Millán (2011) explican que el compromiso organizacional reside en el enlace psicológico entre el trabajador y la organización e identifican dos ideologías: 1) el deseo de continuar en el empleo o 2) la intención de renunciar. De acuerdo con Mitroff y Denton (1999), el compromiso con la empresa puede ser clasificado en tres dimensiones: afectivo, normativo y de continuidad. El compromiso afectivo se desarrolla cuando el personal reconoce el valor y la relevancia de su identidad, y goza del apoyo y respeto de la organización. Dicho compromiso afectivo se fortalece y se manifiesta a través de la interacción social, el reconocimiento social y la actividad diaria del capital social en el lugar de trabajo. El compromiso normativo se desarrolla y es inculcado cuando el personal internaliza las normas y valores universales a través de la socialización y el compromiso del día a día. Finalmente, el compromiso de continuidad enfatiza el reconocimiento de los costos que implica la retención de un empleado o su dimisión.

La renuncia o dimisión se refiere a la separación voluntaria entre el empleado y la organización (Yueran \& Liu, 2015). Las razones para renunciar, de acuerdo con Qazi, Khalid y Shafique (2015), son multifacéticas. Generalmente se ha vinculado al descontento con el salario devengado, poca satisfacción en el trabajo e insatisfacción con el empleo en general. Se estima que la intención de renunciar radica interiormente en el empleado, en las políticas de la organización y en la naturaleza del trabajo. Se relaciona, además, con variables sociodemográficas tales como la edad y el género, la tenencia y pensamientos negativos $\mathrm{y} / \mathrm{o}$ afectivos hacia al puesto que se ocupa (RosarioHernández \& Rovira-Millán, 2011).

Por su parte, Yueran y Liu (2015) señalan que las teorías e investigaciones realizadas sobre la intención de renunciar sugieren que cuando un empleado se encuentra insatisfecho con su trabajo o tiene un nivel bajo de compromiso con la organización, el nivel de la intención de renunciar es mayor. Según Jun-Cheng, Wen-Quan, Zhao-Yi y Jun (2015) existe evidencia fehaciente que indica que el compromiso organizacional yace en el vínculo psicológico del empleado con la organización, el cual puede reducir la intención de renunciar. Sostienen, además, que las teorías sobre la conducta indican que cuando el empleado concibe la intención de renunciar, ya ha estado experimentando síntomas de estrés social. El estrés social puede ser causado por los colegas o compañeros de trabajo afectando las actitudes hacia el trabajo, así como el comportamiento en las relaciones interpersonales.

\section{Espiritualidad en el lugar de trabajo y el compromiso organizacional}

O’Brian (2014) afirma que la inclusión de la espiritualidad en el ámbito laboral tiene unos beneficios en la organización, dado que éstas han demostrado que ayudan en el mejoramiento de la resiliencia, el bienestar y la habilidad para lidiar con los eventos difíciles de la vida. Del mismo modo, establece un vínculo positivo con el compromiso organizacional normativo. Por su parte, Lazar (2010) 
afirma que la inclusión de la espiritualidad en el ambiente de trabajo es fundamental para reducir la insatisfacción laboral y aumentar el compromiso organizacional. El paradigma de la espiritualidad en el lugar de trabajo se ha asociado con la ausencia de conflictos organizacionales, tales como el ausentismo, la rotación, el deseo de renunciar y factores sociodemográficos. En esa misma dirección, Hodge (2001) sostiene que la espiritualidad fortalece la mediación de conflictos y el estrés social producto de los conflictos laborales y es, además, el mecanismo que fortalece las relaciones interpersonales, la comunicación efectiva y la satisfacción laboral.

Nasurdin, Nejati y Mei (2013) encontraron que la edad y el mantenerse en el empleo tuvieron un efecto moderador positivo y significativo en la espiritualidad en el lugar de trabajo y el compromiso organizacional. Mientras, Cambell (2014) encontró una correlación significativa entre la espiritualidad en el ambiente de trabajo y las actitudes positivas hacia el trabajo. De igual forma, la edad de los participantes y la diferencia del género fueron determinantes en la espiritualidad en el ambiente laboral. Por su parte, los estudios de Wainaina, Iravo y Waititu (2014), Malik et al. (2015) y Neubert y Halbesleben (2015), revelaron que existe una correlación positivamente fuerte entre la espiritualidad en el lugar de trabajo y el compromiso organizacional, así como un efecto moderador en el compromiso organizacional. En contraste, Ares-Segarra (2010), Ming-Chia y Chieh-Wen (2013) y Khatri y Gupta (2016) encontraron correlaciones significativamente opuestas entre la espiritualidad en el lugar de trabajo y el compromiso organizacional.
En Puerto Rico, de acuerdo con Pérez (2007) y Rosario-Nieves, Plaza-Montero y Báez-Rivera (2012) son varias las razones que han abonado al interés sobre la espiritualidad en el lugar de trabajo. Estas razones se atribuyen a los cambios en las estructuras organizacionales en los últimos 30 años y a los despidos masivos, siendo los responsables de un efecto negativo en la salud mental y el contexto psicosocial de los trabajadores. El trasfondo del problema de la falta de espiritualidad en el lugar de trabajo estriba en la ausencia del compromiso organizacional, el cual tiene repercusiones en que se produzcan eventos de negligencia, mala práctica, conductas inapropiadas y antiéticas, lo que resulta en litigios y acciones legales (Weld \& Garmon, 2009); así como que coexistan factores tales como la negatividad, el exceso de control y prácticas individualistas (Mitroff \& Denton, 1999), y la presencia de sentimientos de recelo, conductas indecorosas, exceso de jerarquías y traiciones (Marques et al., 2007). Como consecuencia, resulta en el menoscabo de las personas a quienes se les brinda servicio y en cuantiosos costos por litigios legales (Ozbasaran, Ergul, Temel, Gurol, \& Coban, 2011), además de los costos directos e indirectos que se incurre debido a la rotación de empleados y la separación del trabajador del empleo (Hong, 2009).

Por ello, el propósito de la presente investigación fue auscultar la relación entre la espiritualidad en el lugar de trabajo y el compromiso organizacional en un grupo de empleados de un municipio del área oeste de Puerto Rico. Además, conocer el efecto de algunas variables sociodemográficas y la intención de renunciar en la espiritualidad en el lugar de trabajo. Asimismo, comparar los años de servicio, la 
espiritualidad en el ambiente laboral y el compromiso organizacional.

\section{Hipótesis de la investigación}

1. Ha: La espiritualidad en el lugar de trabajo de un grupo de empleados de un municipio del área oeste de Puerto Rico se asocia con el compromiso laboral con un nivel de significancia de $p<0.05$.

2. Ha: La edad, los años de servicio, la preparación académica y la intención de renunciar son variables que predicen la espiritualidad en el ambiente laboral de un grupo de empleados de un municipio del área oeste de Puerto Rico con un nivel de significancia de $\mathrm{p}<0.05$.

3. Ha: Existe diferencia estadísticamente significativa entre los años de servicio, la espiritualidad en el lugar de trabajo y el compromiso organizacional de un grupo de empleados de un municipio del área oeste de Puerto Rico con un nivel de significancia de $\mathrm{p}<0.05$.

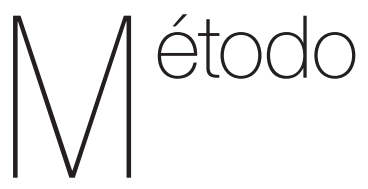

Participantes

En el presente estudio participaron 156 empleados/as del sector público de un municipio del área oeste de Puerto Rico. La muestra fue probabilística, seleccionados/as aleatoriamente. Se les garantizó su voluntariedad, anonimato y el derecho a abandonar su participación cuando lo consideran necesario. En la
Tabla 1 se puede apreciar la distribución de frecuencias de las variables sociodemográficas que manifestaron los participantes del estudio.

Tabla 1.

Distribución de frecuencia de las variables sociodemográficas de la muestra $(n=156)$

\begin{tabular}{|c|c|c|}
\hline Variable & Frecuencia & Porcentaje \\
\hline \multicolumn{3}{|l|}{ Género } \\
\hline Femenino & 84 & 53.8 \\
\hline Masculino & 72 & 46.2 \\
\hline \multicolumn{3}{|l|}{ Edad } \\
\hline $22-31$ & 28 & 17.9 \\
\hline $32-41$ & 48 & 30.8 \\
\hline $42-51$ & 46 & 29.5 \\
\hline $52-61$ & 26 & 16.7 \\
\hline 62 o más & 8 & 5.1 \\
\hline \multicolumn{3}{|l|}{ Preparación académica } \\
\hline Cuarto año Escuela Superior & 40 & 25.6 \\
\hline Créditos universitarios & 40 & 25.6 \\
\hline Bachillerato & 65 & 41.7 \\
\hline Maestría & 3 & 1.9 \\
\hline Doctorado & 2 & 1.3 \\
\hline Grado asociado & 1 & .6 \\
\hline Certificación & 5 & 3.2 \\
\hline \multicolumn{3}{|l|}{ Años de servicio } \\
\hline $1-10$ & 56 & 35.9 \\
\hline $11-20$ & 72 & 46.2 \\
\hline $21-30$ & 21 & 13.5 \\
\hline 310 más & 7 & 4.5 \\
\hline \multicolumn{3}{|l|}{ Probabilidad para renunciar } \\
\hline Muy probable & 25 & 16.0 \\
\hline Algo probable & 58 & 37.2 \\
\hline Nada probable & 73 & 46.8 \\
\hline
\end{tabular}

\section{Instrumentos}

En primer lugar, se utilizó una hoja de datos sociodemográficos, con el cual se les solicitó a los participantes la edad, el sexo, el estado civil, la preparación 
académica y los años de experiencia, entre otras variables, para poder describir la muestra a ser estudiada. E segundo instrumento utilizado fue la Escala de Espiritualidad en el Lugar de Trabajo, desarrollada por Ashmos y Duchon (2000), con el consentimiento de estos. Ésta fue traducida al idioma español y validada para la población puertorriqueña por un grupo de expertos de la Universidad de Puerto Rico del Recinto de Mayagüez. Esta escala fue seleccionada porque es una de las pocas herramientas existentes para medir las actitudes de los empleados hacia espiritualidad en el lugar de trabajo con un nivel apropiado de confiabilidad y cuya validez fue confirmada a través del análisis estadístico de factores, los cuales apoyaron la construcción de las tres sub-escalas (Ares-Segarra, 2010).

La construcción de la escala conceptualiza tres dimensiones de la espiritualidad: individual, unidad de trabajo y organización. De acuerdo con el propósito de esta investigación, se utilizaron solamente las sub-escalas concernientes a la dimensión de la espiritualidad en la unidad de trabajo y la organización, dado que son las que evalúan la percepción del individuo de la espiritualidad en el lugar de trabajo desde la perspectiva del macro ambiente laboral. Este cuestionario se contesta con una escala Likert de cinco puntos, en la que el 1 significa totalmente de acuerdo y 5 totalmente en desacuerdo. La confiabilidad del constructo del cuestionario está apoyada en el método de análisis estadístico de consistencia interna alfa de Cronbach. El mismo tiene una consistencia interna de .93. Por otro lado, las sub-escalas concernientes a la dimensión de espiritualidad de la unidad de trabajo y la organización, tienen un nivel de confiabilidad $\alpha$ de .90 y .93, respectivamente (Ashmos \& Duchon, 2000; Ares-Segarra, 2010). De acuerdo con Pallant (2013), este nivel de confiabilidad significa que la consistencia interna y confiabilidad de la escala es sumamente confiable.

El tercer instrumento utilizado, con el consentimiento de los autores, fue la subescala de Compromiso Moral de la Escala de Compromiso Organizacional desarrollada y validada por Rosario Hernández y Rovira Millán (2002). Este instrumento mide el compromiso moral que sienten las personas hacia la organización para la cual trabajan y la decisión de estos en continuar como parte de la misma. Ésta se contesta mediante una escala Likert de seis puntos, en la que 1 significa totalmente en desacuerdo y 6 totalmente en acuerdo. Su validez fue apoyada a través del análisis estadístico de factores, los cuales apoyaron su estructura con un nivel de confiabilidad alfa de Cronbach de .70. Además, una pregunta del Cuestionario de Calidad de Vida en el Trabajo desarrollado por el Instituto Nacional para la Seguridad y Salud Ocupacional (NIOSH, 2013), con el fin de auscultar la intención de renunciar con opciones de respuestas de tres puntos que van desde muy probable hasta nada probable. La misma fue utilizada por Hong (2009) con el objetivo de correlacionar esta variable con la variable de la espiritualidad en el lugar de trabajo y por Moreno, Cuevas y González (2009) para validar y adaptar el Cuestionario Genérico de Estrés en el Trabajo para la población puertorriqueña, demostrando ser un buen indicador a un nivel de significancia de $p<0.05$ y $p<0.01$. 


\section{Procedimiento}

Se solicitó autorización a la Junta de Revisión Institucional (JRI) de la Pontificia Universidad Católica de Puerto Rico, Recinto de Ponce. Se les informó a los empleados el propósito de la investigación y se les orientó sobre la confidencialidad de la información obtenida, participación voluntaria y sin ninguna recompensa. Se les entregó a aquellos empleados que aceptaron participar una Hoja de Consentimiento Informado, la cual detallaba todos los pormenores de la investigación y las garantías éticas que les cobijan como participantes de una investigación; finalmente, las pruebas fueron administrados a los 156 participantes.

Se utilizó el programa de base de datos Statistical Package for the Social Sciences (SPSS), versión 24.0, para los análisis estadísticos de las variables a investigar y la comprobación de las hipótesis de investigación, los cuales incluyeron la prueba de correlación Pearson (r), regresión múltiple y el análisis de varianza multivariado, por sus siglas en ingles, MANOVA. Conjuntamente, el análisis de varianza unidireccional (ANOVA), para realizar comparaciones múltiples de las medias de los grupos de aquellas variables dependientes que fueron significativas en la prueba MANOVA, a través de la prueba de rango post hoc Scheffé, según recomienda Pallant (2013).

\section{Resultados}

Al observar la relación entre la espiritualidad en el lugar de trabajo y el componente del compromiso organizacional, la espiritualidad en el lugar de trabajo tiene relación significativa, de manera inversa, con el componente del compromiso moral del compromiso organizacional ( $r=-.172, p=.032)$ (ver Tabla 2). Luego de realizar la prueba de suposición para la revisión y cumplimiento de la linealidad, distribución de normalidad de errores y errores no correlacionados (ver Tabla 3), se observa que ninguna variable sociodemográfica tuvo efecto moderador en la percepción de la espiritualidad en el lugar de trabajo a $F(4,151) ; p=1.046$ (ver Tabla 4). En cuanto a diferencias significativas, los años de servicio no parecieron influenciar la espiritualidad en el lugar de trabajo ( $p=.515)$, pero sí, y de manera significativa, el compromiso organizacional normativo $(p=.000)$ (ver Tabla 5).

Tabla 2.

Relación entre la espiritualidad en el lugar de trabajo y el compromiso organizacional

\begin{tabular}{ccc}
\hline & 1 & 2 \\
\hline $\begin{array}{c}\text { Espiritualidad en el } \\
\text { lugar de trabajo }\end{array}$ & - & \\
Compromiso organiza- \\
cional (dimensión del \\
compromiso moral) & $-.172^{*}$ \\
\hline Nota: $p=.032^{*} p<.05$ & \\
\hline
\end{tabular}


Tabla 3.

Media, desviación estándar y correlaciones para la espiritualidad en el lugar de trabajo y las variables predictivas $(N=156)$

\begin{tabular}{ccccccc}
\hline Variables & $\mathbf{M}$ & SD & $\mathbf{1}$ & $\mathbf{2}$ & $\mathbf{3}$ & $\mathbf{4}$ \\
\hline $\begin{array}{c}\text { Espiritualidad en el lugar de trabajo } \\
\text { Variables predictivas }\end{array}$ & 96.76 & 14.73 & .069 & .005 & -.013 & -.095 \\
Edad & & & & & & \\
Años de servicio & 42.13 & 10.86 & & .818 & -.066 & .273 \\
Preparación académica & 12.97 & 8.76 & & -.077 & .222 \\
Intención de renunciar & 2.42 & 1.3 & & & & -.093 \\
\hline
\end{tabular}

Nota: $p<.05$

Tabla 4.

Resumen del análisis de regresión múltiple para las variables predictivas edad, años de experiencia, preparación académica y la intención de renunciar de la espiritualidad en el lugar de trabajo ( $N=156)$

\begin{tabular}{cccc}
\hline Variable & B & SEB & $\beta$ \\
\hline Edad & .310 & .192 & .229 \\
Años de servicio & -.262 & .235 & -.156 \\
Preparación académica & -.251 & .940 & -.022 \\
Intención de renunciar & -2.501 & 1.68 & -.124 \\
Constante & 93.47 & 6.93 & \\
\hline
\end{tabular}

Nota $R^{2}=.001 ; F(4,151)=1.046, p>.05 ;$

Tabla 5.

Resumen de análisis de la varianza multivariado de la espiritualidad en el lugar de trabajo, el compromiso organizacional y los años de servicios

\begin{tabular}{ccccccc}
\hline $\begin{array}{c}\text { Años de } \\
\text { servicios }\end{array}$ & df & SS & MS & $\mathbf{F}$ & $\mathbf{p}$ & $\mathbf{R}^{2}$ \\
\hline $\begin{array}{c}\text { Compromiso } \\
\text { organizacional }\end{array}$ & 166.259 & 3 & 55.420 & 7.944 & .000 & .136 \\
$\begin{array}{c}\text { Espiritualidad } \\
\text { en el lugar } \\
\text { de trabajo }\end{array}$ & 500.686 & 3 & 166.895 & .766 & .515 & .015 \\
\hline Nota: $p<.05$ & & & & & \\
\hline
\end{tabular}

\section{Análisis estadísticos adicionales}

A raíz de los resultados encontrados en el análisis estadístico MANOVA, con respecto a la comparación de la variable del compromiso organizacional y la variable de los años de servicios de los participantes, se realizó la prueba estadística de análisis de la varianza ANOVA. Esta prueba tuvo como objetivo comparar las medias de los años de servicios a través de la prueba de rango post hoc Scheffé, dado que hubo significancia. Los resultados mostraron diferencias 
estadísticamente significativas entre aquellos participantes con uno a veinte años de servicio y los participantes con veintiuno o más años de servicio $(p<.05)$ y el comportamiento organizacional (ver Tabla 5).

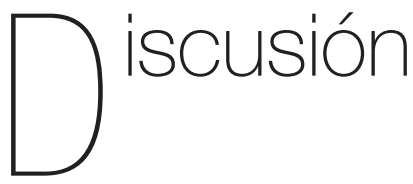

La primera hipótesis del estudio buscaba conocer la relación entre espiritualidad en el lugar de trabajo y el compromiso organizacional en la muestra de estudio. Los resultados revelaron que estas variables están asociadas inversamente la una con la otra a $r=-.172$. Los resultados obtenidos en otros estudios en torno a estas dos variables fueron divergentes con los resultados obtenidos en esta investigación. La mayoría de las investigaciones han demostrado que existe una conexión entre la espiritualidad en el ambiente laboral, el compromiso organizacional, los valores de la organización con el comportamiento y la conducta ética (Duchon \& Plowman, 2005, Fry et al., 2005, Jurkiewicz \& Giacalone, 2004, Maheshwari, 2015; Wainaina et al., 2014).

En contraste, los hallazgos fueron análogos con el estudio de Ares-Segarra (2010), único estudio realizado en Puerto Rico sobre el tema, el cual mostró un alto porcentaje en la percepción de los participantes en torno a cómo la espiritualidad en el lugar de trabajo afecta el comportamiento y en el proceso en la toma de decisiones. Además, muestran que los empleados, en general, están conscientes de cómo el significado del trabajo promueve las actitudes positivas, el futuro de su trabajo y el de la organización. De igual forma, evidencian que los empleados perciben que en sus unidades de trabajo no les importa, ni se preocupan, por su bienestar espiritual. De la misma forma, fue cónsono con el estudio de Khatri y Gupta (2016) y Ming-Chia y Chieh-Wen (2013), cuyos resultados mostraron una correlación significativamente opuesta entre la espiritualidad en el lugar de trabajo y el compromiso organizacional, dado a la percepción negativa de las políticas organizacionales.

De acuerdo con Ashmos-Duchon (2000), esta tendencia es indicativo de que existe una separación entre el empleado y la organización, por ende, es más difícil que la persona pueda percibir la espiritualidad en el lugar de trabajo. Por su parte, Marques et al. (2007) explican que mientras más fuerte sea el factor espiritual en los empleados, habrá más liderazgo, más confianza, mucha más tolerancia, más altruismo y menos susceptibilidad al estrés, resultando que los empleados demuestren compromiso con la organización, un mayor rendimiento y comportamiento ético. Por lo tanto, se infiere la ausencia de espiritualidad en el lugar de trabajo estudiado, así como un compromiso organizacional que se aleja del sistema de valores.

La segunda hipótesis de la investigación buscaba conocer el efecto de las variables de la edad, los años de experiencia, la preparación académica y la intención de renunciar en torno a la espiritualidad en el ambiente laboral. Los resultados muestran que estas variables no tienen efecto moderador o predictivo de la espiritualidad en el lugar de trabajo. Estos resultados fueron divergentes con los estudios de Tejeda (2015), Van der Walt y de Klerk (2014), Nasurdin et al. (2013), 
entre otros, donde mostraron estar relacionados significativamente y tener efecto moderador significativo. El paradigma de la espiritualidad en el ambiente laboral se ha asociado, contrario a los resultados de este estudio, con la ausencia de conflictos organizacionales, tales como el deseo de renunciar y factores sociodemográficos (Hong, 2009; Khatri \& Gupta, 2016; Lazar, 2010). Esto podría indicar la ausencia de espiritualidad en el lugar de trabajo investigado y, al mismo tiempo, la posible presencia de problemas organizacionales. A tales efectos se infiere que podrían existir otras variables que tengan el efecto predictivo o moderador de la espiritualidad en el lugar de trabajo.

La tercera hipótesis de este estudio pretendió comparar los años de experiencia con respecto a la espiritualidad en el lugar de trabajo y el compromiso organizacional. Los resultados revelaron diferencias estadísticamente significativas en cuanto al compromiso organizacional. Sin embargo, no mostraron diferencias estadísticamente significativas con respecto a la espiritualidad en el lugar de trabajo. El análisis post hoc reveló diferencias significativas entre los años de servicio de los participantes; por un lado, estos resultados fueron cónsonos con los resultados obtenidos por Nasurdin et al. (2013). Por otro lado, en cuanto a las diferencias de los años de servicios con respecto a la espiritualidad en el lugar de trabajo fueron similares con el estudio de Van der Walt y de Klerk (2014), estudio el cual coincide con el grupo de 30 a 40 años de servicio con el nivel más alto de espiritualidad, siendo determinantes en la espiritualidad en el ambiente laboral.

Los resultados sugieren que existe una diferencia generacional en cuanto al compromiso organizacional. Las diferencias encontradas entre el compromiso organizacional y los años de servicio constituyen los elementos de acuerdo con el grado en que los objetivos y valores de los empleados están en alineación con los valores y normas de la organización (Fares \& Noordin, 2016). Además, con el enlace psicológico entre el trabajador y la organización y sus dos vertientes: 1) el deseo de continuar en el empleo o 2) la intención de renunciar (Rosario-Hernández \& Rovira-Millán, 2011). Por tal motivo, se infiere el por qué los resultados no presentan diferencias significativas entre los años de servicio y la espiritualidad en el lugar de trabajo, en comparación con el compromiso organizacional, aparentando que la variable de los años de servicio, ligadas a la generación a la cual pertenecen los participantes, constituyen un factor determinante del enlace psicológico entre el trabajador y la organización, ya sea positivo o negativo.

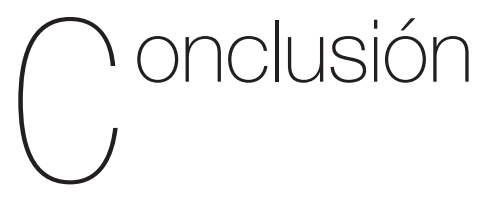

De acuerdo con los resultados obtenidos en esta investigación, se infiere que la relación inversa entre la espiritualidad en el lugar de trabajo y el compromiso organizacional resulta como consecuencia de la percepción negativa de los empleados ante las nuevas políticas organizacionales en Puerto Rico. Estos hallazgos ratifican que el paradigma de la espiritualidad en el lugar de trabajo está ligado estrechamente con el componente psicosocial del individuo (Ashmos \& Duchon, 2000; Mitroff \& Denton, 1999). Los componentes psicosociales, 
de acuerdo con Martín Baró (2012), son aquellos componentes que tienen que ver con los procesos grupales como la toma de decisiones, así como con las acciones e interacción de las personas y grupos. Se considera que la espiritualidad en el lugar de trabajo reprime o abstiene el que se produzcan favores o avances o prácticas discriminatorias, las cuales normalmente están asociadas con las políticas organizacionales (Mitroff \& Denton, 1999). La política organizacional es considerada una combinación compleja de poder, influencia y comportamientos de interés propio. Estos rigen el compromiso organizacional, su ausencia induce a las personas a actuar de modo similar a las carreras de ratas, es decir, a la lucha por la disputa de promociones en el trabajo, salarios más altos y, sobre todo, la seguridad en el empleo, e inherentemente en comportamientos egoístas y destructivos (Khatri \& Gupta, 2016).

\section{Aportaciones a la disciplina}

El concepto de la espiritualidad en el lugar de trabajo, según fundamentado en la revisión de literatura y estudios empíricos expuestos en este estudio, ha demostrado la influencia que esta variable tiene en los empleados con respecto al significado del trabajo, la satisfacción laboral y el compromiso organizacional. De igual manera, afecta favorablemente la organización, dado que se ha asociado positivamente con el desarrollo organizacional, el comportamiento psicosocial y la retención del empleo. La espiritualidad reduce, además, las actitudes negativas y la rotación, fortaleciendo el compromiso laboral y el vínculo con el sistema de valores de la organización, resultando en un modelo de gran envergadura y relevancia para el logro del bienestar del individuo, las organizaciones y la sociedad.

La meta de los Psicólogos Industriales/ Organizacionales, según Aamodt (2013), en el campo del desarrollo y comportamiento organizacional, es incrementar la congruencia entre las estructuras, los procesos, las estrategias, las personas y la cultura de la organización a fin de fortalecer las conductas de los trabajadores y lograr cambios positivos tanto en el micro ambiente como en el macro ambiente. Igualmente, el desarrollo y aplicación de teorías innovadoras que ofrezcan nuevas alternativas para afrontar los retos que enfrentan las organizaciones según los cambios en los contextos políticos, sociales, tecnológicos y económicos. Es importante recalcar que la espiritualidad en el ambiente laboral no constituye la realización de prácticas o imposición de dogmas religiosos en el lugar de trabajo, sino que reconoce que las personas tienen una vida interior que es sustentada a través del significado del trabajo y el sentido de comunidad. Los trabajos que promueven la espiritualidad en su cultura organizacional reconocen que los individuos tienen mente y espíritu (Ashmos \& Duchon, 2000).

Se considera que el presente estudio aporta en lo académico y en la práctica de la psicología industrial organizacional, una herramienta para enfrentar los nuevos retos organizacionales en las políticas públicas, dada la crisis fiscal que enfrenta Puerto Rico. Así como los cambios trascendentales que se están dando en cuanto a la administración de los recursos humanos, tanto en el sector público como el privado, teniendo en cuenta que 
el paradigma de la espiritualidad en el lugar de trabajo conceptualiza el humanismo, el altruismo, las normas éticas y los valores. Además, fomenta el sentido y satisfacción de la vida, los cuales son afectados adversamente debido a los cambios en los contextos económicos, políticos y sociales e inciden en el deterioro del bienestar emocional, mental, conductas maladaptativas y espiritual de los puertorriqueños, los cuales aquejan tanto a la persona como a su entorno en general (Rosario-Nieves et al., 2012).

\section{Recomendaciones para futuras investigaciones}

De acuerdo con los resultados obtenidos para esta investigación, el tema de la espiritualidad en el lugar de trabajo y su relación con el compromiso organizacional se puede considerar un modelo innovador para la psicología organizacional y del trabajo para el desarrollo organizacional y fortalecer el clima laboral. Puerto Rico se encuentra en una era de cambios trascendentales en todos los aspectos concernientes al trabajo, en donde la innovación a través del modelo de la espiritualidad en el lugar de trabajo representa oportunidades para el desarrollo social y el fortalecimiento del compromiso organizacional y por ende el comportamiento organizacional.

La introducción de nuevos estilos o filosofías de visualizar el lugar de trabajo es vital para enfrentar los nuevos retos de competitividad del país, tanto en el sector público como privado. Si se utilizan las bases del comportamiento organizacional, psicología del trabajo y desarrollo organizacional será una herramienta útil para la aplicación de otros estudios que consideren otras variables que puedan afectar o moderar la espiritualidad en el lugar de trabajo y el compromiso normativo organizacional. De igual modo, otros estudios de metodología mixta, los cuales consideren observaciones tales como la comunicación no verbal y entrevistas que ausculten la percepción de la espiritualidad en el lugar de trabajo de las personas y el clima organizacional. Finalmente, el promulgar el concepto de espiritualidad en el lugar de trabajo influye fuertemente en el comportamiento organizacional normativo, la intención de renunciar y los valores éticos.

\section{Limitaciones}

Entre las limitaciones para esta investigación se encontró la dificultad que durante el periodo de la recopilación de los datos se estaba dando la discusión del proyecto de la reforma laboral en el Senado de Puerto Rico. Al mismo tiempo, los empleados del municipio encuestado fueron notificados de reducción de su jornada laboral, demostrando algo de apatía para participar muy en especial los empleados de 26 años o más de servicio. Los cuestionarios por auscultar temas emergentes, los participantes tendían a contestar los cuestionarios de forma ambigua.

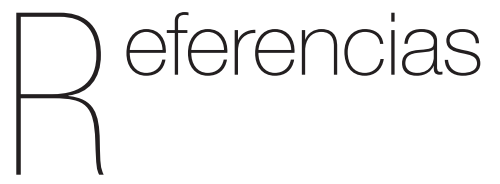

Aamodt, M. G. (2013). Industrial/ organizational psychology: An applied approach (3rd ed.). Belmont, CA: Wadsworth. 
Ares-Segarra, W. (2010). Workplace spirituality: A comparative study (Order No. 1487509). Available from Dissertations \& Theses @ University of Puerto Rico - Mayaguez. (822426802). Retrieved from https://home. uprm.edu/biblioteca/applogin.php?url=/ docview/822426802?accounti $d=28498$

Ashmos, D. P., \& Duchon, D. (2000). Spirituality at work. Journal of Management Inquiry, 9(2), 134.

Belalcázar, S. (2012). Cultura organizacional. Revista Informes Psicológicos, 12, 41-51.

Cambell, J.K. (2014). Workplace spirituality and organizational commotment influence on job performance among academic staff. Journal Pengurusan, 40, 115-123.

Carroll, S. T., Stewart Sicking, J. A., \& Thompson, B. (2014). Sanctification of work: Assessing the role of spirituality in employment attitudes. Mental Health, Religion \& Culture, 17(6), 545-556. doi:10.1 080/13674676.2013.860519

Creswell, J. W. (2014). Research design qualitative, quantitative, and mixed methods approach (4th ed.). Thousand Oaks, California: SAGE Publications, Inc.

Departamento del Trabajo Recursos Humanos del Gobierno de Puerto Rico (2014).

Causales de discrimen en las querellas presentadas en la Unidad Antidiscrimen del Departamento del Trabajo y Recursos Humanos en Puerto Rico. Recuperado de http://www2.pr.gov/agencias/ mujer/Estadisticas/Documents/2015/ Querellas\%20po r\%20Causal\%20 de\%20Discrimen.pdf
Djordjević, D., Petrović, D., Vuković, D., Mihailović, D., \& Dimić, A. (2015). Motivation and job satisfaction of health workers in a specialized health institution in Serbia. Vojnosanitetski Pregled: Military Medical \& Pharmaceutical Journal of Serbia, 72(8), 714-721. doi:10.2298/ VSP131110055D

Duchon, D., \& Plowman, D. A. (2005). Nurturing the spirit at work: Impact on work unit performance. Leadership Quarterly, 16(5), 807-833. doi: 10.1016/j.leaqua. 2005.

07.008

Fachrunnisa, O., Adhiatma, A., \& Mutamimah. (2014). The role of work place spirituality and employee engagement to enhance job satisfaction and performance.

International Journal of Organizational Innovation, 7(1), 15-35.

Fares, D., \& Noordin, K. b. (2016). Islamic Spirituality, Organizational Commitment, and Organizational Citizenship Behavior: A Proposed Conceptual Framework. Middle East Journal of Business, 11(2), 28-37.

French Jr., J. P., \& Kahn, R. L. (1962). A Programmatic Approach to Studying the Industrial Environment and Mental Health. Journal of Social Issues, 18(3), 1-47.

Fry, L. W., Vitucci, S., \& Cedillo, M. (2005). Spiritual leadership and army transformation: Theory, measurement, and establishing a baseline. Leadership Quarterly, 16(5), 835-862. doi:10.1016/j. leaqua.2005.07.012

Goméz- Mejía, L.R. (1992). Diversification, compensation strategy and firm performance. Strategic Management Journal, 13, 121-134. 
Gupta, M., Kumar, V., \& Singh, M. (2014). Creating satisfied employees through workplace spirituality: A study of the private insurance sector in Punjab (I n d i a ). Journal of Business Ethics, 122(1), 79-88. doi:10.1007/s10551-013-1756-5

Heinsohn, D. (2012). Spiritualityin the workplace: An empirical analysis of the moderator effect of demographics on the relationship among spirituality, attitudes, and

behavior at work (Order No. 3502338). Available from ProQuest Central.

(963537033). Retrieved from ht tp s:// home.uprm.edu/biblioteca/applogin. php?url=/docview/963537033?accounti $d=28498$

Hodge, D. (2001). Spirituality assessment: A review of major qualitative methods and a new framework for assessing spirituality. Social Work, 46(3), 203-214.

Hong, Y. J. (2009). The influence of perceived workplace spirituality on job satisfaction, intention to leave, and emotional exhaustion among community mental health center workers in the state of kansas (Order No. 3386587). Available from ProQuest Central. (304907577). Retrieved from ht tps:// home.uprm.edu/biblioteca/applogin. php?url=/docview/304907577?accounti d=28498

Instituto Nacional para la Seguridad y Salud Ocupacional. (2013). Cuestionario de calidad de vida en el trabajo. Recuperado de www.cdc.gov/niosh/ topics/stress/ qwlquest.html

Jun-Cheng, Z., Wen Quan, L., Zhao-Yi, Z., \& Jun, $X$. (2015). Organizational commitment, work engagement, person-supervisor fit, and turnover intention: A total effect

moderation model. Social Behavior \& Personality: An International Journal, 43(10), 1657-1666.

Jurkiewicz, C. L., \& Giacalone, R. A. (2004). A values framework for measuring the impact of workplace spirituality on organizational performance. Journal of

Business Ethics, 49(2), 129-142.

Kazemipour, F., Mohamad Amin, S., \& Pourseidi, B. (2012). Relationship between workplace spirituality and organizational citizenship behavior among nurses through mediation of affective organizational commitment. Journal of Nursing Scholarship, 44(3), 302-310 9p. doi:10.1111/j.1547-5069.2012.01456.x

Khatri, P. P., \& Gupta, P. G. (2016). A Study of Relationship of Perceived Organizational Politics and Spirituality at Workplace in IT and ITES Organizations. Global Journal of Enterprise Information System, 8(1), 1-8.

Kolodinsky, R., Giacalone, R., \& Jurkiewicz, C. (2008). Workplace Values and Outcomes: Exploring Personal, Organizational, and Interactive Workplace Spirituality.

Journal of Business Ethics, 81(2), 465-480. doi:10.1007/s10551-007-9507-0

Lazar, A. (2010). Spirituality and job satisfaction among female Jewish Israeli hospital nurses. Journal of Advanced Nursing, 66(2), 334-344. doi:10.1111/j.1365-

2648.2009.05172.x

Madden, T. (2015). Journeys of purpose: A review of literature about work and spirituality. International Journal of Religion \& Spirituality in Society, 5(4), 69-76 
Maheshwari, N. (2015). Spiritual Intelligence: Occupational Commitment. SCMS Journal of Indian Management, 12(2), 29-38.

Malik, S., Riaz, N., \& Nazir, S. (2015). Personal spirituality and work attitudes among doctors. Journal of Behavioral Sciences, 25(1), 136-149.

Marques, J., Dhiman, S., \& King, R. (2005). Spirituality in the Workplace: Developing an Integral Model and a Comprehensive Definition. Journal of American Academy of Business, Cambridge, 7(1), 81-91.

Marques, J., Dhiman, S., \& King, R. (2007). Spirituality in the workplace. USA:

Personhood Press.

Marschke, E., Preziosi, R., \& Harrington, W. J. (2011). How sales personnel view the relationship between job satisfaction and spirituality in the workplace. Journal of Organizational Culture, Communications \& Conflict, 15(2), 71-110.

Martín Baró, I. (2012). Acción e ideología psicología social desde Centroamérica (15ta ed.). El Salvador: Talleres Gráficos UCA

Ming-Chia, C., \& Chieh-Wen, S. (2013). Workplace spirituality and earnings management motivations. International Journal of Business \& Information, 8(2), 267-298.

Mitroff, I.I., \& Denton, E.A. (1999). A spiritual audit of corporate America. San Francisco, CA: Jossey-Bass Inc.

Moreno, F., \& Godoy, E. (2008). Los nuevos desafíos en la gerencia de los recursos humanos: Calidad de vida laboral. Revista
Daena (International Journal of Good Conscience), 3(2), 1-11.

Moreno, I., Cuevas, M., \& González, J. (2009). Validación y adaptación del cuestionario de calidad de vida en el trabajo (QWLQ) y del cuestionario genérico de estrés en el trabajo (GJSQ). Revista Interamericana de Psicología Organizacional, 28(1), 56-

80.

Nasurdin, A. M., Nejati, M., \& Mei, Y. K. (2013). Workplace spirituality and organizational citizenship behaviour: Exploring gender as a moderator. South African Journal of Business Management,44(1), 61-74.

Neubert, M., \& Halbesleben, K. (2015). Called to commitment: An examination of relationships between spiritual calling, Job Satisfaction, and Organizational Commitment. Journal of Business Ethics, 132(4), 859-872. doi:10.1007/s10551014-2336-z

O'Brian, M. E. (2014). Spirituality in nursing (5th ed.). Burlington, MA: Jones \& Barlett Learning.

Ozbasaran, F., Ergul, S., Temel, A., Gurol, G., \& Coban, A. (2011). Turkish nurses' perceptions of spirituality and spiritual care. Journal of Clinical Nursing, 20(21/22), 31023110. doi:10.1111/j.1365-2702.2011.03778.x

Pallant, J. (2013). SPSS survival manual (5th ed.). China: McGraw Hill.

Pérez, J. (2007). Estudio exploratorio sobre el tema de la espiritualidad en el ambiente laboral. Anales de Psicología, 23(1), 137-146.

Qazi, T. F., Khalid, A., \& Shafique, M. (2015). Contemplating employee retention through 
multidimensional assessment of turnover intentions. Pakistan Journal of Commerce \& Social Sciences, 9(2), 598-613.

Rosario-Hernández, E., \& RoviraMillán, L. V. (2011). La relación entre las manifestaciones de conductas de ciudadanía organizacional con los cinco factores de la personalidad, satisfacción laboral, compromiso organizacional, justicia organizacional y contrato psicológico. Puerto Rican Journal of Psychology/Revista Puertorriqueña de Psicología, 22(2), 72-100.

Rosario-Nieves, I., Plaza-Montero, A., \& Báez-Rivera, A. (2012). La salud mental en Puerto Rico: Una mirada desde las estadísticas y las políticas públicas. Recuperado de /uploads/ publicacionesSalud_mentalpr/files/assets/ basic- html/page3.html

Sheep, M. L. (2004). Nailing down gossamer: A valid measure of the personorganization fit of workplace spirituality. Academy ofManagement Proceedings, B1B6. doi:10.5465/AMBPP.2004.13862520

Tejeda, M. (2015). Exploring the supportive effects of spiritual well-being on job satisfaction given adverse work conditions. Journal of Business Ethics, 131(1), 173-181. doi:10.1007/s10551-014-2269-6
Van der Walt, F., \& de Klerk, J. J. (2014). Workplace spirituality and job satisfaction. International Review of Psychiatry (Abingdon, England), 26(3), 379- 389. doi:10.3109/09540261.2014.908 826

Wainaina, L., Iravo, M., \& Waititu, A. (2014). Workplace spirituality as a determinant of organizational commitment amongst academic staff in the private and public universities in Kenya. International Journal of Academic Research in Business and Social Sciences, 4(12), 280-293. doi: 10.6007/JJARBSS/v4-i12/1362

Weld, K., \& Garmon, B.S. (2009). Concept analysis: Malpractice and modern-day nursing practice. Nursing Forum, 44(1), 2-10. doi:10.1111/j.1744-6198.2009.00121.x

Young, C., \& Koopsen, C. (2011). Spirituality, health, and healing: An integrative approach (2nd ed.). Sudbury, Massachusetts: Jones and Bartlett Publishers.

Yueran, W., \& Liu, L. (2015). Career plateau and turnover intention in Chinese employees: careeranchorasmoderator. SocialBehavior \& Personality: An International Journal, 43(5), 757-766. 
pp •11-30 Rita Azarell Vélez-Alvarado y Roberto González Valles

Informes Psicológicos

30 Vol. 19 No. 1 • Enero-Junio • 2019

ISSN - e: 2422-3271 